\title{
A systematic review of the effectiveness of interventions to improve post-fracture investigation and management of patients at risk of osteoporosis
}

Elizabeth A Little, Martin P Eccles ${ }^{*}$

\begin{abstract}
Background: There is a large quality of care gap for patients with osteoporosis. As a fragility fracture is a strong indicator of underlying osteoporosis, it offers an ideal opportunity to initiate investigation and treatment. However, studies of post-fracture populations document screening and treatment rates below $20 \%$ in most settings. This is despite the fact that bone mineral density (BMD) scans are effective at identifying patients at high risk of fracture, and effective drug treatments are widely available. Effective interventions are required to remedy this incongruity in current practice.

Methods: This study reviewed randomised controlled trials (RCT) involving fully qualified healthcare professionals caring for patients with a fragility fracture in all healthcare settings. Any intervention designed to modify the behaviour of healthcare professionals or implement a service delivery change was considered. The main outcomes were BMD scanning and osteoporosis treatment with anti-resorptive therapy. The electronic databases Medline and Embase were searched from 1994 to June 2010 to identify relevant articles in English. Post-intervention risk differences (RDs) were calculated for the main outcomes and any additional study primary outcomes; the trials were meta-analysed.

Results: A total of 2814 potentially relevant articles were sifted; 18 were assessed in full text. Nine RCTs evaluating ten interventions met the inclusion criteria for the review. All were from North America. Four studies focused on patients with a hip fracture, three on fractures of the wrist/distal forearm, and two included several fracture sites consistent with a fragility fracture. All studies reported positive effects of the intervention for the main study outcomes of BMD scanning and osteoporosis treatment. For BMD scanning the overall risk ratio (95\% Cl) was 2.8 (2.16 to 3.64 ); the RD was $36 \%$ (21\% to $50 \%$ ). For treatment with anti-resorptive therapy the overall risk ratio ( $95 \%$ Cl) was 2.48 (1.92 to 3.2); the RD was $20 \%$ (10\% to $30 \%$ ).

Conclusions: All interventions produced positive effects on BMD scanning and osteoporosis treatment rates postfracture. Despite sizeable increases, investigation and treatment rates remain sub-optimal. Long-term compliance with osteoporosis medications needs to be addressed, as the majority of studies reported treatment rates at sixmonth follow up only. Studies would be more informative if treatment criteria were defined a priori to facilitate understanding of whether patients were being treated appropriately and integrated economic analyses would be helpful for informing policy implementation decisions.
\end{abstract}

\footnotetext{
* Correspondence: martin.eccles@ncl.ac.uk

Institute of Health and Society, Newcastle University, Baddiley-Clark Building,

Richardson Road, Newcastle upon Tyne, NE2 4AX, UK
} 


\section{Background}

Osteoporosis is, 'a progressive systemic skeletal disease characterised by low bone mass and micro-architectural deterioration of bone tissue, with a consequent increase in bone fragility and susceptibility to fracture [1].' Osteoporosis can be diagnosed clinically (vertebral fracture in a 80 year old white female) or defined by a $\mathrm{T}$-score of -2.5 standard deviations or lower on bone mineral density (BMD) scanning. It is well documented that there is a large quality of care gap for patients with this condition [2-11]. This has two main components: firstly a failure to make a diagnosis of osteoporosis, and secondly to manage the condition adequately once the diagnosis has been made.

A fragility fracture is 'a fracture caused by injury that would be insufficient to fracture normal bone: the result of reduced compressive and/or torsional strength of bone' [12]. It is a strong indicator of underlying osteoporosis, and it has been shown that adults who sustain a fracture are over $50 \%$ more likely to have another at a different anatomical site $[13,14]$. Therefore, a first fracture offers an ideal opportunity to initiate investigation and, if indicated, treatment for osteoporosis. However, studies of postfracture populations document screening and treatment rates below $20 \%$ in most settings [5,8-11,15-20]. This is despite the fact that BMD scans are effective at identifying patients at high risk of fracture [21-23], and drug treatments have been shown to significantly reduce the rates of subsequent fragility fractures, even in individuals with advanced bone loss and prevalent fractures [24-30].

Although published evidence-based guidelines exist for the investigation and management of osteoporosis [31-35], the gap between accepted recommendations for osteoporosis care and current practice remains wide. The reasons for this are unclear, although several barriers have been suggested and explored [36-43]. Reported reasons include: lack of consensus as to who is responsible for initiating treatment; lack of awareness by patients and physicians of the treatment guidelines and efficacy of medications for osteoporosis following fragility fracture; and the adverse effects and high costs of medications. Recent studies have shown that $70 \%$ to 90\% of PCPs wish to be more informed about the management of osteoporosis $[39,41,42]$, and with PCPs assuming the prime responsibility for addressing osteoporosis over recent years, this is an ideal setting in which to implement change.

The aim of this review is to assess within randomised controlled trials (RCTs) the effectiveness of interventions to improve the investigation (BMD scanning) and management of osteoporosis (treatment with antiresorptive therapy) in patients following a fragility fracture.

\section{Methods \\ Criteria for considering studies for this review Types of studies}

This review focused on RCTs as they provide the least biased estimate of the effectiveness of an intervention.

\section{Types of participants and settings}

This review focused on fully qualified healthcare professionals of any discipline caring for patients with a fragility fracture. All healthcare settings were included, i.e., community, primary, secondary, and tertiary care.

\section{Types of interventions}

This review focused on any intervention or combination of interventions designed to improve the investigation and management of osteoporosis following fragility fracture by modifying the behaviour of healthcare professionals or implementing a service delivery change, with usual care as comparator.

\section{Outcome measures}

The main review outcomes of interest were BMD scanning and osteoporosis treatment with anti-resorptive therapy. Other outcomes considered were: diagnosis of osteoporosis, prescribing of calcium and vitamin D, and economic variables. A study was required to report on at least one of the main review outcomes to be considered for this review.

\section{Search methods for identification of studies}

The electronic databases Medline and Embase were searched from 1994 (reflecting the introduction of BMD scanning and use of anti-resorptive medications such as the bisphosphonates into practice) to June 2010. The search strategy incorporated the Cochrane RCT sensitivity maximising filter combined with selected $\mathrm{MeSH}$ terms and free text terms related to interventions to improve investigation and management of osteoporosis following fragility fracture (see Additional File 1 for the search strategy used in full). The search was limited to English language articles.

In addition to the electronic searches, the reference lists of relevant studies were hand searched to identify any further relevant studies, and the following were contacted to enquire about any additional published or unpublished data relevant to this review: National Osteoporosis Society (UK), National Osteoporosis Foundation (US), International Osteoporosis Foundation, and experts in the field.

\section{Data collection and analysis \\ Selection of studies}

One review author (EAL) screened all titles and abstracts of retrieved studies in Endnote. If a study met the initial selection criteria or its eligibility could not be 
determined from the title and abstract, the full text was retrieved. Both review authors then independently assessed the full text for inclusion status, and any disagreements were resolved through discussion.

\section{Data extraction and management}

EAL undertook data abstraction of each of the included studies using a modified Cochrane Effective Practice and Organisation of Care (EPOC) Data Collection Checklist. The data extraction form was pilot tested on one included study. Data was extracted on study design, study objectives, participants, instrument reliability and validity, type of interventions, sample size, statistical power, primary and secondary study findings, statistical tests used, and associated statistical and clinical significance. MPE independently assessed the data extracted and conclusions drawn.

\section{Assessment of risk of bias in included studies}

We used The Cochrane Collaboration's tool for assessing risk of bias on six standard criteria: adequate sequence generation, concealment of allocation, blinded or objective assessment of primary outcome(s), adequately addressed incomplete outcome data, free from selective reporting, and free of other risk of bias [44]. We used three additional criteria specified by the EPOC Review Group: similar baseline characteristics, similar baseline outcome measures, and adequate protection against contamination [45]. No studies were excluded because of poor methodological quality.

\section{Measures of treatment effect}

We report the main results for each study in natural units extracted from the results presented in articles. Only the main review outcomes, as well as additional study primary outcomes are reported in full. We had planned to report adjusted risk differences for the review outcomes that adjust for baseline compliance. However, clinicians were unlikely to test those who have been tested or treat those who were already treated for osteoporosis, making the notion of baseline compliance with the review outcomes less meaningful. Therefore, post-intervention risk differences (RD) were calculated and reported instead. For a study to be included in the review, it had to report actual numbers of patients receiving a BMD scan or anti-resorptive treatment for osteoporosis following fragility fracture to enable the post-intervention RD to be calculated (one study was rejected on these grounds). As an overall summary measure of effectiveness, the risk ratio and RDs were calculated using meta-analysis for both of the main outcomes of the review.

\section{Results}

\section{Selection of studies for inclusion}

Figure 1 describes the process from searching to study inclusion. Searches of the electronic databases to June
2010 yielded a total of 2,814 potentially relevant articles (following de-duplication). Following review of titles and abstracts, we obtained 18 articles for assessment in full text. Nine RCTs met the inclusion criteria for the review [46-54], with nine being excluded for the reasons detailed in Figure 1 [55-63]. No additional potential studies were identified through hand-searching reference lists of articles, or by contacting experts in the field or osteoporosis foundations/societies.

\section{Characteristics of study design and setting}

Table 1 describes the included studies. There were eight two-arm RCTs [46,48-54] and one three-arm RCT [47]. Five of the studies were conducted in the US $[46,47,50,53,54]$ and four in Canada $[48,49,51,52]$. Four of the studies were set in tertiary care university hospitals or medical centres $[46,48,53,54]$, with the intervention being targeted at primary care physicians (PCPs) in three of them $[46,48,54]$. Two studies were set solely in primary care $[47,50]$. The remaining three studies were set in hospital $[49,51,52]$ with the PCP being the target of the intervention in two of them [51,52]. The unit of allocation was the patient in seven [46-49,52-54], the physician in one [50], and the family practice in one [51]. It was not possible to produce an overall number of sites and healthcare professionals; the studies included 3,302 patients.

Four studies focused on patients with a hip fracture $[46,48,49,53]$, three on fractures of the wrist/distal forearm [51,52,54], and two included several fracture sites consistent with a fragility fracture $[47,50]$. One study specified that the fracture was a fragility fracture [54], and four that the fracture was low energy/minimal trauma $[46,48,51,53]$, but the remaining four studies did not discriminate by mechanism of injury $[47,49,50,52]$.

\section{Types of intervention}

The intervention (content and method of delivery) and the care delivered to the control groups are described in Table 1. Two of the interventions were directed at the PCP through patient education $[46,48]$, one was an electronic medical record (EMR) reminder sent to the PCP [47], one included an EMR reminder to the PCP plus a patient reminder [47], three incorporated both PCP and patient education [50-52], and three were service delivery changes $[49,53,54]$. Two of the service delivery changes took the responsibility of investigating and treating the osteoporosis out of the hands of the PCP $[49,53]$, and in one the investigation was carried out but the results were sent to the PCP to act on [54].

\section{Development of the intervention}

With regards to the development of the intervention, only two studies reported consulting with the professional 


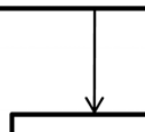

- 1 controlled before and after trial [57]

- 4 no comprehensive subgroup analysis for patients with previous fracture [58-61]

- 1 patient-orientated intervention, no control group [62]

- 1 cost-analysis study [63]

9 randomised controlled trials included in analysis [46-54]

Figure 1 Study Flowchart.

recipients $[49,52]$. Six studies reported the evidence base for the intervention [46,47,49-52]. Consumer involvement was not reported by any of the studies. In four of the studies, the authors report specific barriers to change that the intervention was tailored to address $[46,49,51,53]$.

\section{Risk of bias in included studies}

The risk of bias in included studies is reported in Table 2. Six trials reported adequate sequence generation, four reported adequate concealment of allocation, and four reported either adequately blinded or objective assessment of primary outcome. All studies adequately addressed incomplete outcome data, but only for two studies did it appear that they were free from selective reporting. Seven studies were judged to be free from other biases; one of the other two studies was stopped early, reporting that it was deemed unethical to continue following an interim analysis. It is unclear if this interim analysis was pre-specified. Eight studies had similar baseline characteristics and all had similar baseline outcome measures (for treatment but not for BMD scanning). Three studies were judged to have adequate protection against contamination.

Although producing summary scores or categories across the various risk of bias criteria is not recommended, the results in Table 2 suggest that only one-third of the studies were likely to be at low risk of bias.

\section{Effects of interventions}

Review outcomes and study primary outcomes of included studies are reported in Table 3 . With regards to the main review outcomes of BMD scanning and osteoporosis treatment with anti-resorptive therapy, all studies report positive effects of the interventions. The results are shown in Forest Plots in Figures 2 to 5. For BMD scanning the overall risk ratio $(95 \% \mathrm{CI})$ was 2.8 (2.16 to 3.64 ) and there was a small to medium, nonsignificant amount of heterogeneity $\left(\mathrm{I}^{2} 42 \%\right)$; the RD was $36 \%$ ( $21 \%$ to $50 \%)$. For treatment with anti-resorptive therapy the overall risk ratio $(95 \% \mathrm{CI})$ was 2.48 (1.92 to 3.2$)$ and there was no heterogeneity $\left(\mathrm{I}^{2} 7 \%\right)$; the RD was $20 \%$ (10\% to $30 \%)$. Funnel plots (Additional File 2) suggest some asymmetry, but there are too few studies to formally assess this.

\section{Other outcomes}

Calcium and vitamin D use was reported in four studies $[48,51,52,54]$, but there was only a statistically significant difference between control and intervention group in 
Table 1 Characteristics of included studies

\begin{tabular}{|c|c|c|c|c|c|c|c|c|}
\hline Reference & Setting & Design & $\begin{array}{l}\text { Trial } \\
\text { subjects }\end{array}$ & Inclusions & Exclusions & $\begin{array}{l}\text { Content and method of } \\
\text { delivery of intervention }\end{array}$ & Control group & Comments \\
\hline $\begin{array}{l}\text { Gardner } \\
2005 \text { [46] }\end{array}$ & $\begin{array}{l}\text { One tertiary care } \\
\text { university medical } \\
\text { centre; primary } \\
\text { care; New York, } \\
\text { USA. }\end{array}$ & $\begin{array}{l}\text { Two arm } \\
\text { RCT; patient } \\
\text { randomised. } \\
\text { No power } \\
\text { calculation } \\
\text { reported. } \\
\text { F/U period: } \\
\text { six months. }\end{array}$ & $\begin{array}{l}\text { Clinicians } \\
\text { PCPs* } \\
\text { (further } \\
\text { details not } \\
\text { reported). } \\
\text { Patients } \\
\mathrm{N}=80 \text {. } \\
\text { Mean age: } 82 \\
\text { years. } \\
78 \% \text { female. }\end{array}$ & Low energy hip fracture. & $\begin{array}{l}\text { Antiresorptive medication } \\
\text { use, under } 65 \text { years, } \\
\text { alcoholism, dementia. }\end{array}$ & $\begin{array}{l}\text { Content } \\
15 \text { mins patient } \\
\text { education; five questions } \\
\text { to take to PCP regarding } \\
\text { investigation, diagnosis } \\
\text { and management of } \\
\text { osteoporosis; patient } \\
\text { reminder at six weeks } \\
\text { post-op. } \\
\text { Delivery } \\
\text { Visit by clinical research } \\
\text { coordinator during } \\
\text { hospitalization; printed } \\
\text { copy of questions; phone } \\
\text { call. }\end{array}$ & $\begin{array}{l}\text { Prior to discharge, } \\
\text { patients given two page } \\
\text { pamphlet on fall } \\
\text { prevention based on a } \\
\text { National Osteoporosis } \\
\text { Foundation publication. }\end{array}$ & \\
\hline $\begin{array}{l}\text { Feldstein } \\
2006 \text { [47] }\end{array}$ & $\begin{array}{l}\text { One Pacific } \\
\text { Northwest non- } \\
\text { profit health } \\
\text { maintenance } \\
\text { organization } \\
\text { (HMO) involving } \\
15 \text { primary clinics; } \\
\text { USA. }\end{array}$ & $\begin{array}{l}\text { Three arm } \\
\text { RCT; patient } \\
\text { randomised. } \\
\text { Power } \\
\text { calculation } \\
\text { reported } \\
\text { and } \\
\text { sufficient } \\
\text { numbers } \\
\text { recruited. } \\
\text { F/U period: } \\
\text { six months. }\end{array}$ & $\begin{array}{l}\text { Clinicians } \\
159 \text { PCPs. } \\
\text { Patients } \\
N=327 . \\
\text { Age range = } \\
\text { 50-89 years. } \\
\text { 100\% female. }\end{array}$ & $\begin{array}{l}\text { Individuals aged } 50 \text { to } 89 \\
\text { who had been HMO } \\
\text { members for at least } 12 \\
\text { months and sustained a } \\
\text { study defined fracture } \\
\text { (any clinical fracture } \\
\text { except skull, facial, finger, } \\
\text { toe, ankle or any open } \\
\text { fracture). }\end{array}$ & $\begin{array}{l}\text { Previous BMD scan/ } \\
\text { osteoporosis treatment, } \\
\text { malignancy, chronic renal } \\
\text { failure, organ transplant, } \\
\text { cirrhosis, dementia, men, } \\
\text { nursing home residents, } \\
\text { no address, no primary } \\
\text { care provider, research } \\
\text { centre employees. }\end{array}$ & $\begin{array}{l}\text { Intervention one: } \\
\text { Content } \\
\text { Physician alert and } \\
\text { education. } \\
\text { Delivery } \\
\text { Patient-specific electronic } \\
\text { medical record (EMR) in- } \\
\text { basket message from } \\
\text { chairman of the } \\
\text { osteoporosis quality- } \\
\text { improvement committee; } \\
\text { internal and external } \\
\text { guideline resources; } \\
\text { second message sent at } \\
\text { three months if no } \\
\text { investigation/treatment } \\
\text { carried out. } \\
\text { Intervention two: } \\
\text { Content } \\
\text { Physician alert and } \\
\text { education; patient } \\
\text { reminder and education } \\
\text { copied to PCP. } \\
\text { Delivery } \\
\text { Patient-specific EMR in- } \\
\text { basket message as above } \\
\text { with copy of patient } \\
\text { reminder; printed } \\
\text { educational materials in } \\
\text { advisory letter to patient. }\end{array}$ & $\begin{array}{l}\text { Usual care - if patient is } \\
\text { hospitalized for a fracture, } \\
\text { the PCP receives a copy } \\
\text { of the discharge } \\
\text { summary and the patient } \\
\text { is followed-up by } \\
\text { orthopaedists in a } \\
\text { fracture clinic. }\end{array}$ & $\begin{array}{l}\text { Except for exclusion of } \\
\text { open fractures, no } \\
\text { attempt made to } \\
\text { distinguish between } \\
\text { fractures that resulted } \\
\text { from high force as data } \\
\text { not reliably available } \\
\text { electronically. }\end{array}$ \\
\hline
\end{tabular}


Table 1 Characteristics of included studies (Continued)

\begin{tabular}{|c|c|c|c|c|c|c|c|c|}
\hline $\begin{array}{l}\text { Davis } 2007 \\
\text { [48] }\end{array}$ & $\begin{array}{l}\text { One tertiary care } \\
\text { university hospital; } \\
\text { primary care; } \\
\text { Vancouver, } \\
\text { Canada. }\end{array}$ & $\begin{array}{l}\text { Two arm } \\
\text { RCT; patient } \\
\text { randomised. } \\
\text { Power } \\
\text { calculation } \\
\text { reported. } \\
\text { F/U period: } \\
\text { six months. }\end{array}$ & $\begin{array}{l}\text { Clinicians } \\
\text { PCPs (further } \\
\text { details not } \\
\text { reported). } \\
\text { Patients } \\
\mathrm{N}=48 . \\
\text { Mean age: } \\
82.6 \text { years } \\
\text { (control), } 80.4 \\
\text { years. } \\
\text { (intervention) } \\
71 \% \text { female. }\end{array}$ & $\begin{array}{l}\text { All women and men } \geq \\
60 \text { years residing in } \\
\text { Vancouver admitted with } \\
\text { a minimal trauma hip } \\
\text { fracture. }\end{array}$ & $\begin{array}{l}\text { On osteoporosis } \\
\text { treatment, dementia/ } \\
\text { cognitive impairment, } \\
\text { unable to communicate } \\
\text { in English, severe medical } \\
\text { pathology (e.g. cancer, } \\
\text { chronic renal failure). }\end{array}$ & $\begin{array}{l}\text { Content } \\
\text { Patient education and } \\
\text { advice to visit PCP for } \\
\text { further investigation; } \\
\text { physician alert. } \\
\text { Delivery } \\
\text { Osteoporosis information; } \\
\text { letter for patient to take } \\
\text { to PCP from orthopaedic } \\
\text { surgeon. }\end{array}$ & $\begin{array}{l}\text { Usual care for the } \\
\text { fracture and a phone call } \\
\text { at three months (general } \\
\text { health inquiry) and } 6 \\
\text { months to determine } \\
\text { whether osteoporosis } \\
\text { investigation and } \\
\text { treatment had occurred. }\end{array}$ & $\begin{array}{l}\text { Minimal trauma defined } \\
\text { as falling from a } \\
\text { standing height or less. } \\
\text { Power calculation } \\
\text { required sample size of } \\
44.48 \text { subjects recruited } \\
\text { but } 20 \text { in control group } \\
\text { and } 28 \text { in intervention } \\
\text { group. No explanation } \\
\text { for uneven numbers } \\
\text { between groups } \\
\text { reported. }\end{array}$ \\
\hline $\begin{array}{l}\text { Majumdar } \\
2007 \text { [49] }\end{array}$ & $\begin{array}{l}\text { Three hospitals in } \\
\text { Capital Health } \\
\text { System; } \\
\text { Edmonton, } \\
\text { Alberta, Canada. }\end{array}$ & $\begin{array}{l}\text { Two arm } \\
\text { RCT; patient } \\
\text { randomised. } \\
\text { Power } \\
\text { calculation } \\
\text { reported } \\
\text { and } \\
\text { sufficient } \\
\text { numbers } \\
\text { recruited. } \\
\text { F/U period: } \\
\text { six months. }\end{array}$ & $\begin{array}{l}\text { Clinicians } \\
\text { One case- } \\
\text { manager } \\
\text { (registered } \\
\text { nurse), one } \\
\text { study } \\
\text { physician. } \\
\text { Patients } \\
\mathrm{N}=220 \text {. } \\
\text { Median age: } \\
74 \text { years. } \\
60 \% \text { female. }\end{array}$ & $\begin{array}{l}\text { Community-dwelling } \\
\text { patients } \geq 50 \text { years with } \\
\text { hip fracture undergoing } \\
\text { surgical fixation with no } \\
\text { contraindications to } \\
\text { bisphosphonates and } \\
\text { able to provide (or have } \\
\text { a proxy provide) } \\
\text { informed consent. }\end{array}$ & $\begin{array}{l}\text { Delirium, dementia, on } \\
\text { osteoporosis treatment, } \\
\text { pathologic fractures, } \\
\text { patients in nursing } \\
\text { homes or long-term care } \\
\text { facilities. }\end{array}$ & $\begin{array}{l}\text { Content } \\
\text { Usual care; patient } \\
\text { education; outpatient } \\
\text { BMD test; prescription for } \\
\text { bisphosphonates for } \\
\text { patients with low bone } \\
\text { mass; communication to } \\
\text { PCPs regarding results } \\
\text { and treatment plans. } \\
\text { Delivery } \\
\text { Case-manager - provided } \\
\text { one-on-one counselling; } \\
\text { arranged BMD test; } \\
\text { obtained prescription } \\
\text { from study physician to } \\
\text { be dispensed by local } \\
\text { community pharmacy. }\end{array}$ & $\begin{array}{l}\text { Study personnel provided } \\
\text { counselling about fall } \\
\text { prevention and intake of } \\
\text { calcium and vitamin D; } \\
\text { educational materials } \\
\text { from osteoporosis } \\
\text { Canada provided and } \\
\text { patients asked to discuss } \\
\text { the material with their } \\
\text { PCP. }\end{array}$ & $\begin{array}{l}\text { Canadian guidelines } \\
\text { recommended } \\
\text { pharmacologic } \\
\text { osteoporosis therapy in } \\
\text { patients with a fragility } \\
\text { fracture after age } 50 \\
\text { years or menopause } \\
\text { and a BMD T score } \leq \\
-1.5 \text {. } \\
\text { Patients in control } \\
\text { group received more } \\
\text { education and study- } \\
\text { related attention than } \\
\text { true usual care as } \\
\text { practiced in most } \\
\text { Canadian or US centres. }\end{array}$ \\
\hline $\begin{array}{l}\text { Solomon } \\
2007[50]\end{array}$ & $\begin{array}{l}\text { Primary care } \\
\text { (patients all } \\
\text { beneficiaries of } \\
\text { HBCBSN }{ }^{\Delta} \text { health } \\
\text { care insurer); New } \\
\text { Jersey, USA. }\end{array}$ & $\begin{array}{l}\text { Two arm } \\
\text { cluster RCT; } \\
\text { physician } \\
\text { randomised } \\
\text { (provided at } \\
\text { least four } \\
\text { patients per } \\
\text { physician). } \\
\text { Analysis } \\
\text { adjusted for } \\
\text { clustering. } \\
\text { No power } \\
\text { calculation } \\
\text { reported. F/ } \\
\text { U period: } 10 \\
\text { months. }\end{array}$ & $\begin{array}{l}\text { Clinicians } \\
434 \text { PCPs. } \\
\text { Mean age: } 50 \\
\text { years. 17\% } \\
\text { female. } \\
\text { Patients } \\
N=1973 \\
\text { (229 with } \\
\text { fractures). } \\
\text { Mean age: } 69 \\
\text { years } \\
\text { (control), } 68 \\
\text { years } \\
\text { (intervention). } \\
\text { 92\% female. }\end{array}$ & $\begin{array}{l}\text { HBCBSNJ beneficiaries } \\
\text { who had at least two } \\
\text { years of enrolment and a } \\
\text { prescription drug benefit; } \\
\text { required to have filed at } \\
\text { least one prescription } \\
\text { claim in each of the two } \\
\text { baseline years; age } \geq 45 \\
\text { years; prior fracture of } \\
\text { hip, spine, forearm or } \\
\text { humerus. }\end{array}$ & $\begin{array}{l}\text { Previous BMD scan or } \\
\text { prescription for } \\
\text { osteoporosis medication } \\
\text { during baseline } 26 \\
\text { months; patients whose } \\
\text { PCP had < four eligible } \\
\text { patients at risk for } \\
\text { osteoporosis. }\end{array}$ & $\begin{array}{l}\text { Content } \\
\text { Physician education; } \\
\text { physician alert; patient } \\
\text { education; patient } \\
\text { invitation to attend BMD } \\
\text { scan. } \\
\text { Delivery } \\
\text { One-on-one educational } \\
\text { visit with PCP conducted } \\
\text { by specially trained } \\
\text { pharmacists who work } \\
\text { with HBCBSNJ as } \\
\text { physician educators; } \\
\text { continuing medical } \\
\text { education (CME) } \\
\text { program; list of at-risk } \\
\text { patients given to PCP } \\
\text { and discussed at } \\
\text { meeting; printed } \\
\text { educational materials and } \\
\text { letter from HBCBSNJ to } \\
\text { patient; automated } \\
\text { phone call invitation for } \\
\text { BMD scan. }\end{array}$ & $\begin{array}{l}\text { No description, assumed } \\
\text { usual care. }\end{array}$ & $\begin{array}{l}\text { Figures for subgroup of } \\
\text { patients with prior } \\
\text { fracture included in } \\
\text { review taken from } \\
\text { baseline characteristics } \\
\text { of wider study } \\
\text { population. } \\
\text { The study paid for } \\
\text { doctors to apply for } \\
\text { CME credit if they } \\
\text { completed a post-visit } \\
\text { test. }\end{array}$ \\
\hline
\end{tabular}


Table 1 Characteristics of included studies (Continued)

\begin{tabular}{|c|c|c|c|c|c|c|c|}
\hline $\begin{array}{l}\text { Cranney } \\
2008 \text { [51] }\end{array}$ & $\begin{array}{l}\text { Emergency } \\
\text { departments or } \\
\text { fracture clinics of } \\
\text { five hospitals (two } \\
\text { of which were } \\
\text { teaching } \\
\text { hospitals); } 119 \\
\text { primary care } \\
\text { practices; Ontario, } \\
\text { Canada. }\end{array}$ & $\begin{array}{l}\text { Two arm } \\
\text { cluster RCT; } \\
\text { family } \\
\text { practice } \\
\text { randomised. } \\
\text { Analysis } \\
\text { adjusted for } \\
\text { clustering. } \\
\text { Power } \\
\text { calculation } \\
\text { reported } \\
\text { and } \\
\text { sufficient } \\
\text { numbers } \\
\text { recruited. } \\
\text { F/U period: } \\
\text { six months. }\end{array}$ & $\begin{array}{l}\text { Clinicians } \\
174 \text { PCPs. } \\
55 \% \text { female. } \\
54 \text { practiced } \\
\text { in rural } \\
\text { settings. } \\
\text { Patients } \\
\mathrm{N}=270 . \\
\text { Mean age: } \\
69.8 \text { years } \\
\text { (control), } 68.1 \\
\text { years } \\
\text { (intervention). } \\
\text { 100\% female. }\end{array}$ & $\begin{array}{l}\text { Family practices in } \\
\text { Kingston, Ontario and the } \\
\text { surrounding southeastern } \\
\text { Ontario region drawn } \\
\text { from the Canadian } \\
\text { Medical Association } \\
\text { directory; post- } \\
\text { menopausal women who } \\
\text { had sustained a wrist } \\
\text { fracture (confirmed by x- } \\
\text { ray). }\end{array}$ & $\begin{array}{l}\text { Osteoporosis medication } \\
\text { use, traumatic wrist } \\
\text { fracture, unable to } \\
\text { communicate in English } \\
\text { or unable to give } \\
\text { consent. }\end{array}$ & $\begin{array}{l}\text { Content } \\
\text { Physician alert; physician } \\
\text { education; patient } \\
\text { reminder recommending } \\
\text { F/U visit with PCP; } \\
\text { patient education. } \\
\text { Delivery } \\
\text { Personalised letter mailed } \\
\text { to PCP by research } \\
\text { coordinator at two weeks } \\
\text { and two months post- } \\
\text { fracture; two page } \\
\text { educational tool and } \\
\text { treatment algorithm from } \\
\text { Osteoporosis Canada's } \\
\text { clinical practice } \\
\text { guidelines; mailed } \\
\text { patient reminder letter at } \\
\text { two weeks and two } \\
\text { months post-fracture; } \\
\text { educational booklet. }\end{array}$ & $\begin{array}{l}\text { Usual care. Patients and } \\
\text { PCPs were not sent any } \\
\text { communication until trial } \\
\text { completed. }\end{array}$ \\
\hline $\begin{array}{l}\text { Majumdar } \\
2008 \text { [52] }\end{array}$ & $\begin{array}{l}\text { Two emergency } \\
\text { departments and } \\
\text { two fracture } \\
\text { clinics, Capital } \\
\text { Health; primary } \\
\text { care; Edmonton, } \\
\text { Alberta, Canada. }\end{array}$ & $\begin{array}{l}\text { Two arm } \\
\text { RCT; patient } \\
\text { randomised. } \\
\text { Power } \\
\text { calculation } \\
\text { reported } \\
\text { and } \\
\text { sufficient } \\
\text { numbers } \\
\text { recruited. } \\
\text { F/U period: } \\
\text { six months. }\end{array}$ & $\begin{array}{l}\text { Clinicians } \\
266 \text { PCPs. } \\
\text { Patients } \\
\mathrm{N}=272 . \\
\text { Median age: } \\
60 \text { years. } \\
77 \% \text { female. }\end{array}$ & $\begin{array}{l}\text { Age } \geq 50 \text { years and any } \\
\text { distal forearm fracture, } \\
\text { regardless of cause. }\end{array}$ & $\begin{array}{l}\text { Bisphosphonate use, } \\
\text { unable or unwilling to } \\
\text { provide informed } \\
\text { consent, no fixed address, } \\
\text { residing outside Capital } \\
\text { Health region, residing in } \\
\text { a long-term care facility. }\end{array}$ & $\begin{array}{l}\text { Content } \\
\text { Patient education and } \\
\text { advice to discuss } \\
\text { osteoporosis with PCP; } \\
\text { patient-specific reminders } \\
\text { to PCPs; physician } \\
\text { education. } \\
\text { Delivery } \\
\text { Phone counselling } \\
\text { session to patients by } \\
\text { experienced registered } \\
\text { nurse; physician reminder } \\
\text { sent by fax or mail; } \\
\text { evidence based } \\
\text { treatment guidelines } \\
\text { endorsed by opinion } \\
\text { leaders sent to PCPs. }\end{array}$ & $\begin{array}{l}\text { Given Osteoporosis } \\
\text { Canada pamphlet and } \\
\text { encouraged to discuss } \\
\text { with PCP, second copy } \\
\text { mailed to patient. PCPs } \\
\text { routinely notified that } \\
\text { their patients had been } \\
\text { treated for a wrist } \\
\text { fracture and informed of } \\
\text { F/U plans and } \\
\text { appointment. }\end{array}$ \\
\hline
\end{tabular}




\begin{tabular}{|c|c|c|c|c|c|c|c|c|}
\hline $\begin{array}{l}\text { Miki } 2008 \\
{[53]}\end{array}$ & $\begin{array}{l}\text { One tertiary care } \\
\text { university medical } \\
\text { centre, inpatient } \\
\text { and outpatient } \\
\text { clinic; Connecticut, } \\
\text { USA. }\end{array}$ & $\begin{array}{l}\text { Two arm } \\
\text { RCT; patient } \\
\text { randomised. } \\
\text { Power } \\
\text { calculation } \\
\text { reported. } \\
\text { F/U period: } \\
\text { six months }\end{array}$ & $\begin{array}{l}\text { Clinicians } \\
\text { One male } \\
\text { orthopaedic } \\
\text { surgeon. } \\
\\
\text { Patients } \\
\mathrm{N}=62 . \\
\text { Mean age: } \\
79.2 \text { years. } \\
71 \% \text { female. }\end{array}$ & $\begin{array}{l}\text { All English-speaking } \\
\text { patients admitted with } \\
\text { low-energy hip fracture. }\end{array}$ & $\begin{array}{l}\text { Osteoporosis medication } \\
\text { use, pathologic fracture. }\end{array}$ & $\begin{array}{l}\text { Content } \\
\text { Patient education; } \\
\text { osteoporosis evaluation; } \\
\text { calcium and vitamin D } \\
\text { commenced; patient } \\
\text { review and } \\
\text { bisphosphonate } \\
\text { commenced as } \\
\text { appropriate; monitoring } \\
\text { of adherence to } \\
\text { medication and } \\
\text { complications; transfer of } \\
\text { responsibility for } \\
\text { medication adherence } \\
\text { and patient management } \\
\text { to PCP after six months. } \\
\text { Delivery } \\
15 \text { mins education to } \\
\text { patient and families } \\
\text { whilst in hospital from } \\
\text { one of the investigators; } \\
\text { inpatient blood tests and } \\
\text { BMD scan; F/U } \\
\text { outpatient orthopaedic } \\
\text { clinic appointment } \\
\text { between two weeks and } \\
\text { one month post-op; } \\
\text { phone call to patient or } \\
\text { clinic visit at two and six } \\
\text { months. }\end{array}$ & $\begin{array}{l}15 \text { mins education on hip } \\
\text { fractures, fracture } \\
\text { prevention and } \\
\text { osteoporosis from one of } \\
\text { the investigators; advised } \\
\text { to see PCP for } \\
\text { osteoporosis evaluation; } \\
\text { commenced on calcium } \\
\text { and vitamin D. }\end{array}$ & $\begin{array}{l}\text { Trial stopped following } \\
\text { interim analysis before } \\
\text { pre-defined sample size } \\
\text { reached due to ethical } \\
\text { reasons. }\end{array}$ \\
\hline $\begin{array}{l}\text { Rozental } \\
2008[54]\end{array}$ & $\begin{array}{l}\text { One university } \\
\text { tertiary care } \\
\text { centre, } \\
\text { orthopaedic } \\
\text { outpatient clinic; } \\
\text { primary care; } \\
\text { Boston, USA. }\end{array}$ & $\begin{array}{l}\text { Two arm } \\
\text { RCT; patient } \\
\text { randomised. } \\
\text { Power } \\
\text { calculation } \\
\text { reported } \\
\text { and } \\
\text { sufficient } \\
\text { numbers } \\
\text { recruited. } \\
\text { F/U period: } \\
\text { six months. }\end{array}$ & $\begin{array}{l}\text { Clinicians } \\
\text { PCPs, } \\
\text { orthopaedic } \\
\text { surgeons } \\
\text { (further } \\
\text { details not } \\
\text { reported). } \\
\text { Patients } \\
\mathrm{N}=50 \text {. } \\
\text { Mean age: } 65 \\
\text { years. } \\
\text { 92\% female }\end{array}$ & $\begin{array}{l}\text { Women }>50 \text { years or } \\
\text { men }>65 \text { years; fragility } \\
\text { fracture of distal part of } \\
\text { radius. }\end{array}$ & $\begin{array}{l}\text { High energy trauma, } \\
\text { BMD scan within two } \\
\text { years of fracture, current } \\
\text { HRT or antiresorptive } \\
\text { medication use. }\end{array}$ & $\begin{array}{l}\text { Intervention one: } \\
\text { Content } \\
\text { BMD scan with results } \\
\text { forwarded to PCP. } \\
\text { Delivery } \\
\text { Scan ordered by } \\
\text { orthopaedic surgeon } \\
\text { during patient's initial } \\
\text { office visit for fracture } \\
\text { care; results forwarded } \\
\text { by mail and email to } \\
\text { PCP. }\end{array}$ & $\begin{array}{l}\text { Intervention two: } \\
\text { Letter sent by email and } \\
\text { mail to PCP outlining } \\
\text { national guidelines for } \\
\text { evaluating and treating } \\
\text { osteoporosis after fragility } \\
\text { fracture; the guidelines } \\
\text { included ordering a BMD } \\
\text { scan within six months of } \\
\text { injury. }\end{array}$ & $\begin{array}{l}\text { Intervention two } \\
\text { considered to be close } \\
\text { enough to usual care to } \\
\text { use as a control group. } \\
\text { Fragility fracture defined } \\
\text { as those resulting from } \\
\text { a standing height or } \\
\text { less. }\end{array}$ \\
\hline
\end{tabular}


Table 2 Risk of bias of included studies

\begin{tabular}{|c|c|c|c|c|c|c|c|c|c|}
\hline Reference & $\begin{array}{l}\text { Adequate } \\
\text { sequence } \\
\text { generation }\end{array}$ & $\begin{array}{l}\text { Concealment } \\
\text { of allocation }\end{array}$ & $\begin{array}{l}\text { Blinded or } \\
\text { objective } \\
\text { assessment of } \\
\text { primary } \\
\text { outcome(s) }\end{array}$ & $\begin{array}{l}\text { Adequately } \\
\text { addressed } \\
\text { incomplete } \\
\text { outcome } \\
\text { data }\end{array}$ & $\begin{array}{l}\text { Free from } \\
\text { selective } \\
\text { reporting }\end{array}$ & Free from other risk of bias & $\begin{array}{l}\text { Similar } \\
\text { baseline } \\
\text { characteristics }\end{array}$ & $\begin{array}{l}\text { Similar } \\
\text { baseline } \\
\text { outcome } \\
\text { measures }\end{array}$ & $\begin{array}{l}\text { Adequate protection against } \\
\text { contamination }\end{array}$ \\
\hline $\begin{array}{l}\text { Gardner } \\
2005[46]\end{array}$ & Yes & Unclear & Unclear & Yes & $\begin{array}{l}\text { Unclear - protocol } \\
\text { not published; trial } \\
\text { registration } \\
\text { number not } \\
\text { reported. }\end{array}$ & $\begin{array}{l}\text { Unclear - only approx. } 20 \% \text { of } \\
\text { patients approached were included } \\
\text { in the study. } 40 \% \text { of patients } \\
\text { deemed eligible declined to enter } \\
\text { study. }\end{array}$ & $\begin{array}{l}\text { Unclear - not } \\
\text { reported. }\end{array}$ & $\begin{array}{l}\text { Yes for } \\
\text { treatment; } \\
\text { unclear for } \\
\text { BMD } \\
\text { scanning. }\end{array}$ & $\begin{array}{l}\text { Unclear - patient randomised, } \\
\text { PCPs not reported. }\end{array}$ \\
\hline $\begin{array}{l}\text { Feldstein } \\
2006[47]\end{array}$ & Yes & Yes & Yes & Yes & $\begin{array}{l}\text { Unclear - protocol } \\
\text { not published; trial } \\
\text { registration } \\
\text { number not } \\
\text { reported. }\end{array}$ & Yes & Yes & Yes & $\begin{array}{l}\text { Unclear - patient randomised: } \\
15 \text { primary care clinics involved } \\
\text { with } 159 \text { PCPs, average one to } \\
\text { three patients per PCP. }\end{array}$ \\
\hline $\begin{array}{l}\text { Davis } 2007 \\
\text { [48] }\end{array}$ & Yes & No & No & Yes & $\begin{array}{l}\text { Unclear - protocol } \\
\text { not published; trial } \\
\text { registration } \\
\text { number not } \\
\text { reported. }\end{array}$ & Yes & Yes & $\begin{array}{l}\text { Yes for } \\
\text { treatment; } \\
\text { unclear for } \\
\text { BMD } \\
\text { scanning. }\end{array}$ & $\begin{array}{l}\text { Unclear - patient randomised, } \\
\text { PCPs not reported. }\end{array}$ \\
\hline $\begin{array}{l}\text { Majumdar } \\
2007 \text { [49] }\end{array}$ & Yes & Yes & Yes & Yes & $\begin{array}{l}\text { Yes for primary } \\
\text { outcome; no for } \\
\text { secondary } \\
\text { outcomes. }\end{array}$ & Yes & Yes & $\begin{array}{l}\text { Yes for } \\
\text { treatment; } \\
\text { unclear for } \\
\text { BMD } \\
\text { scanning. }\end{array}$ & $\begin{array}{l}\text { Unclear - possibility of } \\
\text { contamination if control and } \\
\text { intervention patients on ward } \\
\text { at same time. }\end{array}$ \\
\hline $\begin{array}{l}\text { Solomon } \\
2007[50]\end{array}$ & Unclear & Unclear & Unclear & Yes & $\begin{array}{l}\text { Unclear - protocol } \\
\text { not published; trial } \\
\text { registration } \\
\text { number not } \\
\text { reported. }\end{array}$ & Yes & Yes & Yes & $\begin{array}{l}\text { Unclear - physician } \\
\text { randomised but practices in } \\
\text { which they worked not } \\
\text { reported on. }\end{array}$ \\
\hline $\begin{array}{l}\text { Cranney } \\
2008[51]\end{array}$ & Yes & Yes & Yes & Yes & $\begin{array}{l}\text { Unclear - protocol } \\
\text { not published; trial } \\
\text { registration } \\
\text { number not } \\
\text { reported. }\end{array}$ & Yes & Yes & Yes & Yes \\
\hline $\begin{array}{l}\text { Majumdar } \\
2008[52]\end{array}$ & Yes & Yes & Yes & Yes & Yes & Yes & Yes & $\begin{array}{l}\text { Yes for } \\
\text { treatment; } \\
\text { unclear for } \\
\text { BMD } \\
\text { scanning. }\end{array}$ & Yes \\
\hline $\begin{array}{l}\text { Miki } 2008 \\
{[53]}\end{array}$ & Unclear & Unclear & No & Yes & $\begin{array}{l}\text { Unclear - protocol } \\
\text { not published; trial } \\
\text { registration } \\
\text { number not } \\
\text { reported. }\end{array}$ & $\begin{array}{l}\text { Unclear - stopped early as deemed } \\
\text { unethical to continue following } \\
\text { interim analysis. }\end{array}$ & Yes & Yes & Yes \\
\hline $\begin{array}{l}\text { Rozental } \\
2008[54]\end{array}$ & Unclear & Unclear & Unclear & Yes & $\begin{array}{l}\text { Unclear - protocol } \\
\text { not published; trial } \\
\text { registration } \\
\text { number not } \\
\text { reported. }\end{array}$ & Yes & Yes & $\begin{array}{l}\text { Yes for } \\
\text { treatment; } \\
\text { unclear for } \\
\text { BMD } \\
\text { scanning. }\end{array}$ & $\begin{array}{l}\text { Unclear - patient randomised, } \\
\text { PCPs not reported. }\end{array}$ \\
\hline
\end{tabular}


Table 3 Reported study outcomes

\begin{tabular}{|c|c|c|c|c|c|c|}
\hline \multirow{2}{*}{$\begin{array}{l}\text { Reference } \\
\text { Gardner } \\
2005 \text { [46] }\end{array}$} & & \multicolumn{3}{|c|}{ Reported study outcomes } & \multirow{2}{*}{$\begin{array}{l}\text { Osteoporosis medication use } \\
\text { Drugs used }\end{array}$} & \multirow[t]{2}{*}{ Comments } \\
\hline & & Control & Intervention & $\begin{array}{l}\text { Post- } \\
\text { intervention } \\
\mathrm{RD}^{\Delta}\end{array}$ & & \\
\hline & & N (\%) & N (\%) & (\%) & Bisphosphonates. & \\
\hline & BMD scan & $6 / 36(17)$ & $12 / 36(33)$ & 17 & Data source for drug utilisation & \\
\hline & $\begin{array}{l}\text { Osteoporosis } \\
\text { treatment }\end{array}$ & $6 / 36(17)$ & 10/36 (28) & 11 & Patient self-report. & \\
\hline \multirow[t]{8}{*}{$\begin{array}{l}\text { Feldstein } \\
2006[47]\end{array}$} & & Control & Intervention & $\begin{array}{l}\text { Post- } \\
\text { intervention } \\
\text { RD }\end{array}$ & Drugs used & $\begin{array}{l}\text { Secondary outcomes included regular physical activity, total caloric expenditure, } \\
\text { total calcium intake and patient satisfaction. }\end{array}$ \\
\hline & & N (\%) & N (\%) & (\%) & $\begin{array}{l}\text { Bisphosphonate, calcitonin, selective } \\
\text { estrogen receptor modulator, } \\
\text { estrogen medication. }\end{array}$ & \\
\hline & EMR & & & & Data source for drug utilisation & \\
\hline & BMD scan & $2 / 101(2)$ & $40 / 101(40)^{*}$ & 38 & $\begin{array}{l}\text { Electronically from outpatient } \\
\text { pharmacy system. }\end{array}$ & \\
\hline & $\begin{array}{l}\text { Osteoporosis } \\
\text { treatment }\end{array}$ & $5 / 101(5)$ & $28 / 101(28)^{*}$ & 23 & & $\begin{array}{l}\text { No significant differences between the EMR and the EMR + patient reminder arm } \\
\text { with respect to BMD scanning and osteoporosis treatment. }\end{array}$ \\
\hline & $\begin{array}{l}\text { EMR + } \\
\text { patient } \\
\text { reminder }\end{array}$ & & & & & \\
\hline & BMD scan & 2/101 (2) & $36 / 109(33)^{*}$ & 31 & & \\
\hline & $\begin{array}{l}\text { Osteoporosis } \\
\text { treatment }\end{array}$ & $5 / 101(5)$ & $22 / 109(20)^{*}$ & 15 & & \\
\hline \multirow[t]{6}{*}{$\begin{array}{l}\text { Davis } 2007 \\
{[48]}\end{array}$} & & Control & Intervention & $\begin{array}{l}\text { Post- } \\
\text { intervention } \\
\text { RD }\end{array}$ & Drugs used & $\begin{array}{l}4 / 20(20 \%) \text { of the control group and } 11 / 28(39 \%) \text { of the intervention group } \\
\text { received a diagnosis of osteoporosis but this difference was not significant. }\end{array}$ \\
\hline & & N (\%) & N (\%) & (\%) & Bisphosphonates. & \\
\hline & BMD scan & $0 / 20(0)$ & $8 / 28(29)^{*}$ & 29 & Data source for drug utilisation & \\
\hline & $\begin{array}{l}\text { Osteoporosis } \\
\text { treatment }\end{array}$ & 0/20 (0) & $15 / 28(54)^{*}$ & 54 & Patient self-report. & \\
\hline & $\begin{array}{l}\text { Calcium }+ \\
\text { vitamin D }\end{array}$ & $6 / 20(30)$ & 11/28 (39) & 9 & & \\
\hline & $\begin{array}{l}\text { Exercise } \\
\text { prescription }\end{array}$ & 0/20 (0) & $9 / 28(32)^{*}$ & 32 & & \\
\hline $\begin{array}{l}\text { Majumdar } \\
2007[49]\end{array}$ & & Control & Intervention & $\begin{array}{l}\text { Post- } \\
\text { intervention } \\
\text { RD }\end{array}$ & Drugs used & $\begin{array}{l}\text { Secondary outcomes included "appropriate care" (BMD testing with treatment if } \\
\text { bone mass low), recurrent fractures, admissions to hospital and death. }\end{array}$ \\
\hline
\end{tabular}


Table 3 Reported study outcomes (Continued)

\begin{tabular}{|c|c|c|c|c|c|c|}
\hline & & N (\%) & N (\%) & (\%) & $\begin{array}{l}\text { Bisphosphonates - alendronate or } \\
\text { risedronate. }\end{array}$ & $\begin{array}{l}\text { Of } 120 \text { who underwent BMD testing, } 25(21 \%) \text { did not have low bone mass. Of the } \\
95 \text { patients with low bone mass, } 41(43 \%) \text { had a T score at hip or spine between } \\
-1.5 \text { and }-2.5 \text {, and } 54(57 \%) \text { had a T score of } \leq-2.5 \text {. }\end{array}$ \\
\hline & BMD scan & $32 / 110$ (29) & $88 / 110(80)^{*}$ & 51 & Data source for drug utilisation & \\
\hline & $\begin{array}{l}\text { Osteoporosis } \\
\text { treatment }\end{array}$ & 24/110 (22) & $56 / 110(51)^{*}$ & 29 & Not reported. & \\
\hline \multirow[t]{4}{*}{$\begin{array}{l}\text { Solomon } \\
2007[50]\end{array}$} & & Control & Intervention & $\begin{array}{l}\text { Post- } \\
\text { intervention } \\
\text { RD }\end{array}$ & Drugs used & $\begin{array}{l}\text { Only results adjusted for baseline characteristics significant, unadjusted results } \\
\text { insignificant. }\end{array}$ \\
\hline & & N (\%) & N (\%) & (\%) & $\begin{array}{l}\text { HRT, calcitonin, raloxifene, } \\
\text { bisphosphonates, teriparatide. }\end{array}$ & \\
\hline & BMD scan & 4/95 (4) & $11 / 134(8)^{*}$ & 4 & Data source for drug utilisation & \\
\hline & $\begin{array}{l}\text { Osteoporosis } \\
\text { treatment }\end{array}$ & 1/95 (1) & $6 / 134(4)$ & 3 & Health-care utilisation data. & \\
\hline \multirow[t]{4}{*}{$\begin{array}{l}\text { Cranney } \\
2008[51]\end{array}$} & & Control & Intervention & $\begin{array}{l}\text { Post- } \\
\text { intervention } \\
\text { RD }\end{array}$ & Drugs used & $\begin{array}{l}38 / 141(27 \%) \text { of control patients and } 43 / 120(36 \%) \text { of intervention patients received } \\
\text { calcium or vitamin D. This difference was not statistically significant. }\end{array}$ \\
\hline & & N (\%) & N (\%) & (\%) & $\begin{array}{l}\text { One patient raloxifene, } 49 \\
\text { bisphosphonates. }\end{array}$ & $\begin{array}{l}\text { Results for osteoporosis treatment reported for all patients randomised (270) } \\
\text { whereas BMD scanning and calcium or vitamin D only reported for those } \\
\text { completing follow up (261). }\end{array}$ \\
\hline & BMD scan & $36 / 141(26)$ & $64 / 120(53)^{*}$ & 28 & Data source for drug utilisation & $\begin{array}{l}\text { Secondary outcomes included discussion with PCP regarding osteoporosis and } \\
\text { changes in the participant's knowledge of osteoporosis. }\end{array}$ \\
\hline & $\begin{array}{l}\text { Osteoporosis } \\
\text { treatment }\end{array}$ & 15/145 (10) & $35 / 125(28)^{*}$ & 18 & Patient self-report. & $\begin{array}{l}\text { Although baseline BMD scanning was reported, the ARD cannot be calculated as } \\
\text { the numbers at baseline were different to those included in the analysis. }\end{array}$ \\
\hline \multirow[t]{4}{*}{$\begin{array}{l}\text { Majumdar } \\
2008[52]\end{array}$} & & Control & Intervention & $\begin{array}{l}\text { Post- } \\
\text { intervention } \\
\text { RD }\end{array}$ & Drugs used & $\begin{array}{l}\text { 58/135 (43\%) of control patients and 91/137 (66\%) of intervention patients } \\
\text { received calcium and vitamin D. This difference was statistically significant. }\end{array}$ \\
\hline & & N (\%) & N (\%) & (\%) & Bisphosphonates. & $\begin{array}{l}\text { Results adjusted for acid peptic disease, osteoarthritis, current smoking, calcium } \\
\text { and vitamin D use as significant differences found between intervention and } \\
\text { control groups for these } 5 \text { variables. }\end{array}$ \\
\hline & BMD scan & 24/135 (18) & $71 / 137(52)^{*}$ & 34 & Data source for drug utilisation & Secondary outcomes included "appropriate care" and quality of life. \\
\hline & $\begin{array}{l}\text { Osteoporosis } \\
\text { treatment }\end{array}$ & 10/135 (7) & $30 / 137(22)^{*}$ & 14 & $\begin{array}{l}\text { Patient self-report confirmed } \\
\text { through dispensing records of local } \\
\text { pharmacies. }\end{array}$ & $\begin{array}{l}\text { Of the } 95 \text { patients who underwent BMD testing, } 27(28 \%) \text { had normal bone mass, } \\
49(52 \%) \text { had osteopenia (T score }-1.0 \text { to }-2.5) \text {, and } 19(20 \%) \text { had osteoporosis }(T \\
\text { score } \leq-2.5) \text { at either the hip or spine. }\end{array}$ \\
\hline \multirow[t]{2}{*}{$\begin{array}{l}\text { Miki } 2008 \\
{[53]}\end{array}$} & & Control & Intervention & $\begin{array}{l}\text { Post- } \\
\text { intervention } \\
\text { RD }\end{array}$ & Drugs used & No p-value given for difference in BMD scanning between groups. \\
\hline & & N (\%) & N (\%) & $(\%)$ & $\begin{array}{l}\text { One patient calcitonin nasal spray, } \\
21 \text { bisphosphonates. }\end{array}$ & $\begin{array}{l}\text { For those starting osteoporosis treatment the post-intervention RD was } 44 \% \text { but } \\
\text { one patient in the control group and five in the intervention group stopped } \\
\text { before six months. }\end{array}$ \\
\hline
\end{tabular}


Table 3 Reported study outcomes (Continued)

\begin{tabular}{|c|c|c|c|c|c|c|}
\hline & BMD scan & $7 / 24(29)$ & $26 / 26(100)$ & 71 & Data source for drug utilisation & $\begin{array}{l}\text { In the intervention group, } 38 \% \text { of those receiving treatment for osteoporosis had } \\
\text { at least one T score of less than }-2.5 \text {. }\end{array}$ \\
\hline & \multirow[t]{2}{*}{$\begin{array}{l}\text { Osteoporosis } \\
\text { treatment }\end{array}$} & \multirow[t]{2}{*}{$7 / 24(29)$} & \multirow[t]{2}{*}{$15 / 26(58)^{*}$} & \multirow[t]{2}{*}{29} & \multirow[t]{2}{*}{ Patient self-report. } & $\begin{array}{l}\text { Although baseline BMD scanning was reported, the ARD cannot be calculated as } \\
\text { the numbers at baseline were different to those included in the analysis. }\end{array}$ \\
\hline & & & & & & Secondary outcomes included new fracture during the six month follow up period. \\
\hline \multirow[t]{5}{*}{$\begin{array}{l}\text { Rozental } \\
2008[54]\end{array}$} & & $\begin{array}{l}\text { Control } \\
\text { (Intervention } \\
\text { two) }\end{array}$ & $\begin{array}{l}\text { Intervention } \\
\text { One }\end{array}$ & $\begin{array}{l}\text { Post- } \\
\text { intervention } \\
\text { RD }\end{array}$ & Drugs used & $\begin{array}{l}\text { Intervention two sufficiently close to usual care as to be considered as the control } \\
\text { group. }\end{array}$ \\
\hline & & N (\%) & N (\%) & (\%) & $\begin{array}{l}\text { One teriparatide, one calcitonin, } 11 \\
\text { bisphosphonates. }\end{array}$ & \\
\hline & BMD scan & $7 / 23(30)$ & $25 / 27(93)^{*}$ & 62 & Data source for drug utilisation & $\begin{array}{l}4 / 23(17 \%) \text { of control patients and } 15 / 27(56 \%) \text { of intervention patients received } \\
\text { calcium and vitamin D. This difference was not statistically significant. }\end{array}$ \\
\hline & $\begin{array}{l}\text { Osteoporosis } \\
\text { treatment }\end{array}$ & $5 / 23(22)$ & $8 / 27(30)$ & 8 & $\begin{array}{l}\text { Patient self-report and review of } \\
\text { medical records. }\end{array}$ & $\begin{array}{l}\text { Calcium and vitamin D counted as osteoporosis treatment in original paper so } \\
\text { post-intervention RD reported here substantially less ( } 8 \% \text { c.f. } 48 \%) \text {. There was a } \\
\text { significant difference between groups when calcium and vitamin D were included } \\
\text { as osteoporosis treatment but no p-values reported with these excluded. }\end{array}$ \\
\hline & $\begin{array}{l}\text { Treatment } \\
\text { discussed } \\
\text { with PCP }\end{array}$ & $8 / 23(35)$ & $24 / 27(89)^{*}$ & 54 & & $\begin{array}{l}2 / 23(9 \%) \text { of patients in the control group and } 9 / 27(33 \%) \text { of patients in the } \\
\text { intervention group received a diagnosis of osteoporosis. }\end{array}$ \\
\hline
\end{tabular}

*statistically significant difference between groups $(p<0.05)$.

$\triangle \mathrm{RD}=$ risk difference. 


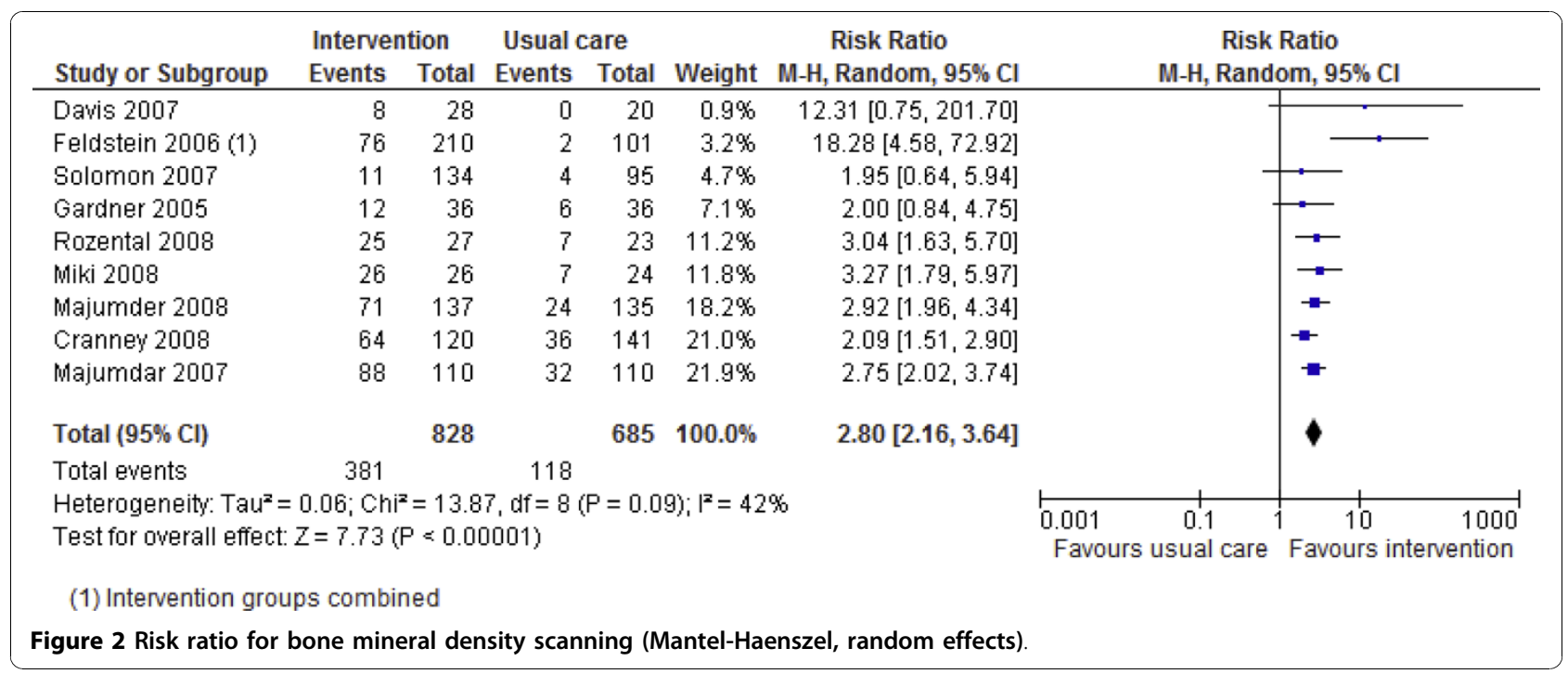

one (43\% versus 66\%) [52]. Two studies reported rates of osteoporosis diagnosis but did not specify what constituted this diagnosis [48,54]. One study reported the percentage of patients undergoing BMD scan given a diagnosis of osteoporosis ( $T$ score $\leq-2.5$ ) [52]. None of the studies reported concurrently economic outcomes, but one trial group [49] subsequently published a separate cost analysis of a case manager intervention that suggested that the intervention was cost saving [63]. Two studies reported on 'appropriate care' [49,52].

Two studies reported primary outcomes other than those addressed by this review [48,54]. One looked at whether or not participants were prescribed exercise as recommended by the 2002 Canadian Medical Association Osteoporosis Clinical Practice Guidelines [48]. They found a statistically significant difference between control and intervention groups with a post-intervention RD of $32 \%$. The other study looked at whether or not osteoporosis had been discussed with the PCP and also found a statistically significant difference between intervention and control groups with a post-intervention $\mathrm{RD}$ of $54 \%$ [54].

\section{Discussion}

We reviewed nine RCTs assessing the effectiveness of a variety of interventions to improve the investigation and management of osteoporosis following fragility fracture. For both of the review main outcomes, BMD scanning and osteoporosis treatment with anti-resorptive therapy, all studies reported a positive effect of the intervention with an overall $36 \%$ absolute increase in scanning rates and a $20 \%$ absolute increase in treatment rates. Such

\begin{tabular}{|c|c|c|c|c|c|c|c|c|}
\hline \multirow[b]{2}{*}{ Study or Subgroup } & \multicolumn{2}{|c|}{ Intervention } & \multicolumn{2}{|c|}{ Usual care } & \multirow[b]{2}{*}{ Weight } & \multirow{2}{*}{$\begin{array}{c}\text { Risk Ratio } \\
\text { M-H, Random, } 95 \% \mathrm{Cl}\end{array}$} & \multirow{2}{*}{\multicolumn{2}{|c|}{$\begin{array}{c}\text { Risk Ratio } \\
\text { M-H, Random, } 95 \% \mathrm{Cl}\end{array}$}} \\
\hline & Events & Total & Events & Total & & & & \\
\hline Davis 2007 & 15 & 28 & 0 & 20 & $0.8 \%$ & $22.45[1.42,354.54]$ & & $\longrightarrow$ \\
\hline Solomon 2007 & 6 & 134 & 1 & 95 & $1.5 \%$ & $4.25[0.52,34.76]$ & & \\
\hline Rozental 2008 & 8 & 27 & 5 & 23 & $6.6 \%$ & $1.36[0.52,3.59]$ & - & - \\
\hline Gardner 2005 & 10 & 36 & 6 & 36 & $7.6 \%$ & $1.67[0.68,4.10]$ & & $-\infty$ \\
\hline Feldstein 2006 (1) & 50 & 210 & 5 & 101 & $7.8 \%$ & $4.81[1.98,11.69]$ & & $\longrightarrow$ \\
\hline Miki 2008 & 15 & 26 & 7 & 24 & $12.0 \%$ & $1.98[0.98,4.00]$ & & -- \\
\hline Majumder 2008 & 30 & 137 & 10 & 135 & $13.0 \%$ & $2.96[1.51,5.81]$ & & $\rightarrow-$ \\
\hline Cranney 2008 & 35 & 125 & 15 & 145 & $18.5 \%$ & $2.71[1.55,4.72]$ & & $\rightarrow$ \\
\hline Majumdar 2007 & 56 & 110 & 24 & 110 & $32.3 \%$ & $2.33[1.57,3.48]$ & & $\rightarrow$ \\
\hline Total $(95 \% \mathrm{Cl})$ & & 833 & & 689 & $100.0 \%$ & $2.48[1.92,3.20]$ & & $\boldsymbol{\gamma}$ \\
\hline Total events & 225 & & 73 & & & & & \\
\hline \multicolumn{6}{|c|}{$\begin{array}{l}\text { Heterogeneity: } \text { Tau }^{2}=0.01 ; \mathrm{Chi}^{2}=8.58, \mathrm{df}=8(\mathrm{P}=0.38) ; \mathrm{I}^{2}=7 \% \\
\text { Test for overall effect: } Z=7.01(\mathrm{P}<0.00001)\end{array}$} & & $\begin{array}{cc}0.002 & 0.1 \\
\text { Favours usual care }\end{array}$ & $\begin{array}{c}10 \\
10 \\
\text { Favours intervention }\end{array}$ \\
\hline \multicolumn{9}{|c|}{ (1) Intervention groups combined } \\
\hline Figure 3 Risk ratio for & anti-resor & tive $d$ & ug trea & $\mathbf{\tau}$ & 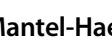 & iszel, random effects). & & \\
\hline
\end{tabular}




\begin{tabular}{|c|c|c|c|c|c|c|c|c|}
\hline \multirow[b]{2}{*}{ Study or Subgroup } & \multicolumn{2}{|c|}{ Intervention } & \multicolumn{2}{|c|}{ Usual care } & \multirow[b]{2}{*}{ Weight } & \multirow{2}{*}{$\begin{array}{c}\text { Risk Difference } \\
\text { M-H, Random, } 95 \% \mathrm{Cl}\end{array}$} & \multirow{2}{*}{\multicolumn{2}{|c|}{$\begin{array}{c}\text { Risk Difference } \\
\text { M-H, Random, } 95 \% \mathrm{Cl}\end{array}$}} \\
\hline & Events & Total & Events & Total & & & & \\
\hline Rozental 2008 & 25 & 27 & 7 & 23 & $9.8 \%$ & $0.62[0.41,0.83]$ & & 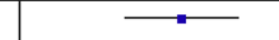 \\
\hline Gardner 2005 & 12 & 36 & 6 & 36 & $10.2 \%$ & $0.17[-0.03,0.36]$ & & \\
\hline Miki 2008 & 26 & 26 & 7 & 24 & $10.3 \%$ & $0.71[0.52,0.90]$ & & \\
\hline Davis 2007 & 8 & 28 & 0 & 20 & $10.5 \%$ & $0.29[0.11,0.46]$ & & \\
\hline Cranney 2008 & 64 & 120 & 36 & 141 & $11.6 \%$ & $0.28[0.16,0.39]$ & & $\rightarrow$ \\
\hline Majumdar 2007 & 88 & 110 & 32 & 110 & $11.6 \%$ & $0.51[0.40,0.62]$ & & $\longrightarrow$ \\
\hline Majumder 2008 & 71 & 137 & 24 & 135 & $11.7 \%$ & $0.34[0.23,0.45]$ & & $\rightarrow$ \\
\hline Feldstein 2006 (1) & 76 & 210 & 2 & 101 & $12.1 \%$ & $0.34[0.27,0.41]$ & & $\rightarrow$ \\
\hline Solomon 2007 & 11 & 134 & 4 & 95 & $12.2 \%$ & $0.04[-0.02,0.10]$ & & 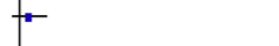 \\
\hline Total $(95 \% \mathrm{Cl})$ & & 828 & & 685 & $100.0 \%$ & $0.36[0.21,0.50]$ & & \\
\hline Total events & 381 & & 118 & & & & & \\
\hline \multicolumn{7}{|c|}{$\begin{array}{l}\text { Heterogeneity: } \text { Tau }^{2}=0.04 ; \text { Chi }^{2}=118.48, d f=8(P<0.00001) ;\left.\right|^{2}=93 \% \\
\text { Test for overall effect: } Z=4.85(P=0.00001)\end{array}$} & $\begin{array}{l}-0.5-0.25 \\
\text { Favours usual care }\end{array}$ & $\begin{array}{lll}0 & 0.25 & 0.5 \\
0 & \text { Favours intervention }\end{array}$ \\
\hline \multicolumn{9}{|c|}{ (1) Intervention groups combined } \\
\hline Figure 4 Risk differen & for bon & nine & I densi & $\operatorname{can}$ & ( & & & \\
\hline
\end{tabular}

effects are clearly important. These results are broadly in agreement with other recent reviews [64] though this had a slightly different focus (patients at risk of osteoporosis) and so included different studies.

Whilst all studies reported positive effects, they have a number of constraints that limit their informativeness. All of the studies were conducted in North America. This may limit their generalisability to other countries and healthcare systems. Although we pooled the results of the studies (reflecting their homogeneity of patient groups and settings), there were still a range of types of interventions and it is not possible to say that any one intervention was more effective than any other. With relatively low levels of control group performance, the results suggest that any intervention is likely to be more effective than usual care; it is not clear what would happen in situations with higher rates of baseline performance. In relation to this, it is relevant to point out that this is a relatively recent body of literature with the oldest trial reporting in 2005. In future it is likely that studies will be addressing improved levels of baseline performance. In some studies it was not clear whether or not treatment was appropriately linked to BMD scanning result, with at least one study apparently reporting treatment on BMD scan values that would not result in treatment in the UK NHS [53]. Whilst it would be expected that most patients should receive a BMD scan post-fracture, the majority of studies failed to report treatment criteria a priori making it difficult to interpret treatment rates. One trial group reported an outcome of guideline concordant 'appropriate care' [49,52]. This was defined as a BMD test performed and osteoporosis

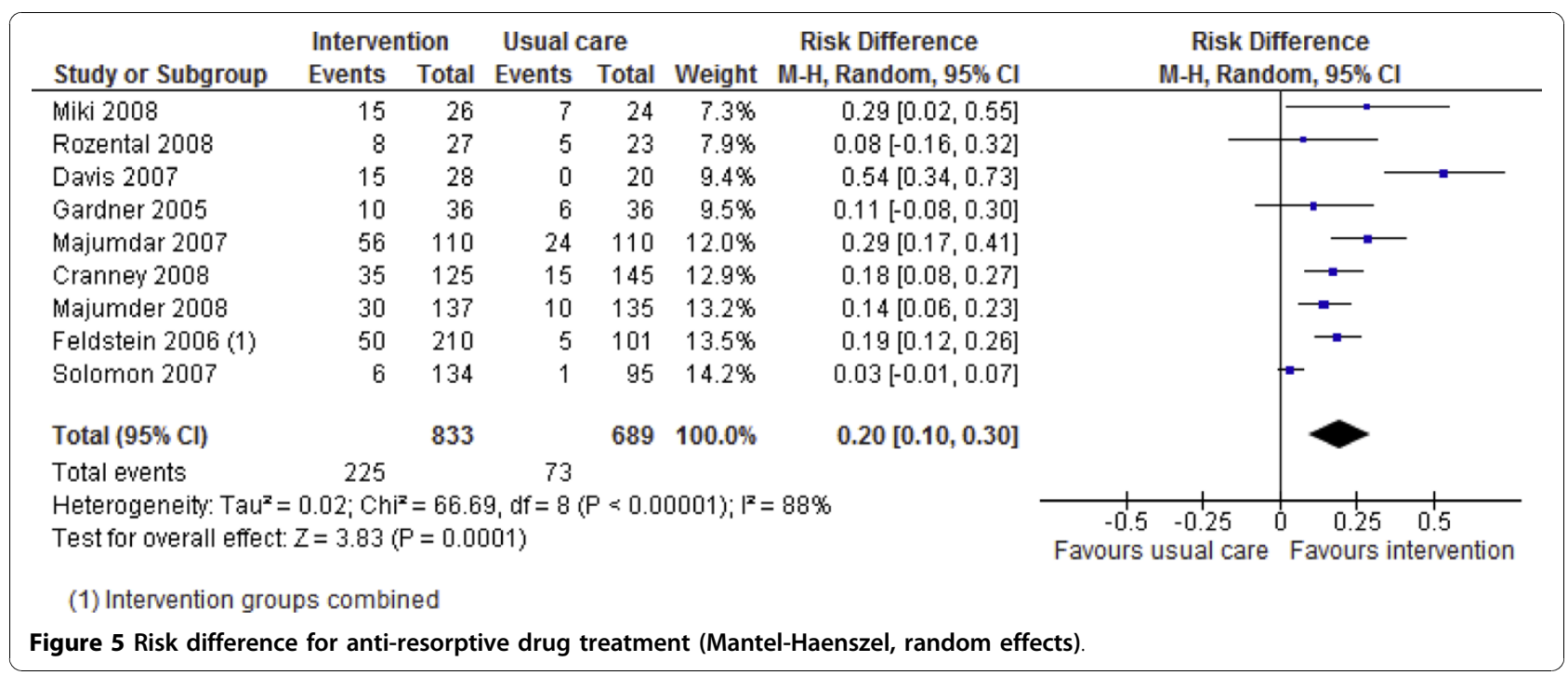


treatment provided to those with low bone mass; they then defined low bone mass according to current guidelines. This maximises our understanding of the data by considering those patients who were appropriately not treated. In the 2007 study, the osteoporosis treatment rate in the intervention group was $51 \%$, yet the rate of 'appropriate care' was $67 \%$ [49]. Thus, $16 \%$ of patients did not receive osteoporosis treatment following BMD scan because they were not eligible for it. Nevertheless, this study also highlights the fact that despite the overall positive effects of the interventions, none of the studies produced maximal rates of investigation and treatment. In this particular study, $33 \%$ of patients did not receive appropriate care.

All of the studies reported treatment rates at six months follow-up, except for one that extended to 10 months [50]. Long-term compliance with osteoporosis medications is not addressed and is something that will need to be considered in future studies.

We assessed all studies for their risk of bias, and five of the nine included studies had multiple unknown criteria in Table $2[46,48,50,53,54]$ It is unclear whether or not these studies were actually at an increased risk of bias or if this assessment was a consequence of poor reporting; however, they tended to be smaller and to report more uncertain results. It is not possible to exclude publication bias. The funnel plots suggested the possible absence of larger, less positive studies, but given the number of included trials this can only be a subjective judgement.

The rationale for the interventions used was often unclear. While four studies reported that the intervention was tailored to identified barriers $[46,49,51,53]$, the other five did not. In addition, from some of the descriptions given it was difficult to extract sufficient detail to be confident that the interventions were being described in a way that would make them replicable $[46-48,50,51,53]$. It was also difficult to disentangle what the investigators felt was the content of their intervention (the active ingredients; e.g., persuasive communication) from the method that they chose to deliver it (printed educational materials). Such distinction is important in order to promote greater clarity in the description of interventions. Recent reporting guidelines have suggested that this will constrain scientific replication and limit the subsequent introduction of successful interventions [65].

Investigators should be considering the economic implications of their interventions and do not, on the basis of this review appear to be doing so; no study concurrently reported an integrated economic analysis of the intervention. However, one trial group subsequently reported an economic analysis of their case-manager intervention that suggested the intervention was cost saving [63].
The review had some limitations. Only articles published in English were considered and only two electronic databases were searched (Medline and Embase). However, it is reassuring that examination outside of the review of Web of Science, OVID Evidence Based Reviews and Cochrane (to June 2010) by one of this manuscript's reviewers identified no additional eligible studies (S. Majumdar, personal communication). Rather than having full duplication of all activities, one author (EAL) sifted the results of the search for included studies, although both authors assessed the eligibility of the 18 articles in which the full text was retrieved. One author (EAL) abstracted data from the included studies; this was checked by the second author.

\section{Summary}

All interventions demonstrated a positive effect on BMD scanning and osteoporosis treatment post-fracture, regardless of healthcare setting, patient population, and type of intervention. Despite this, only one of the studies reported maximal investigation rates (all patients investigated) and none reported maximal treatment rates. Follow-up did not extend beyond 10 months in any of the studies; the issue of long-term compliance with osteoporosis medications will need to be addressed in future studies. To aid interpretation of results, study authors should report treatment criteria a priori and a measure of appropriate care. Integrated economic analyses would be helpful when considering widespread implementation.

\section{Additional material}

Additional file 1: Search Strategy.
Additional file 2: Funnel plot of outcome: Bone Mineral Density
scanning, Mantel-Haenszel random effects model; Funnel plot of
outcome: Osteoporosis treatment, Mantel-Haenszel, random effects
model.

Conflicts of interests

MPE is Co-Editor in Chief of Implementation Science. All editorial decisions on this manuscript were made by another editor.

\section{Acknowledgements}

We are grateful to Fiona Beyer for advice on the search strategy and citation handling. EAL was supported by intramural funding from Northumbria Vocational Training Scheme for General Practice. MPE received intramural support from Newcastle University.

Authors' contributions

MPE conceived the idea of the review. EAL wrote and conducted the searches and sifted the results. Both authors assessed the included and excluded studies. EAL led the writing of the manuscript. Both authors approved the final version.

Received: 7 August 2010 Accepted: 22 October 2010 Published: 22 October 2010 


\section{References}

1. Assessment of fracture risk and its application to screening for postmenopausal osteoporosis: Report of a WHO Study Group. World Health Organization Technical Report Series 1994, 843:1-129.

2. Giangregorio L, Papaioannou A, Cranney A, Zytaruk N, Adachi JD: Fragility fractures and the osteoporosis care gap: an international phenomenon Seminars in Arthritis \& Rheumatism 2006, 35(5):293-305.

3. Elliot-Gibson V, Bogoch ER, Jamal SA, Beaton DE: Practice patterns in the diagnosis and treatment of osteoporosis after a fragility fracture: a systematic review. Osteoporosis International 2004, 15(10):767-778.

4. Freedman KB, Kaplan FS, Bilker WB, Strom BL, Lowe RA: Treatment of osteoporosis: are physicians missing an opportunity? Journal of Bone \& Joint Surgery - American Volume 2000, 82-A(8):1063-1070.

5. Kamel HK, Hussain MS, Tariq S, Perry HM, Morley JE: Failure to diagnose and treat osteoporosis in elderly patients hospitalized with hip fracture. American Journal of Medicine 2000, 109(4):326-328.

6. Colon-Emeric C, Yballe L, Sloane R, Pieper CF, Lyles KW: Expert physician recommendations and current practice patterns for evaluating and treating men with osteoporotic hip fracture. Journal of the American Geriatrics Society 2000, 48(10):1261-1263.

7. Gehlbach SH, Bigelow C, Heimisdottir M, May S, Walker M, Kirkwood JR: Recognition of vertebral fracture in a clinical setting. Osteoporosis International 2000, 11(7):577-582.

8. Andrade SE, Majumdar SR, Chan KA, Buist DS, Go AS, Goodman M, Smith DH, Platt R, Gurwitz JH, Andrade SE, et al: Low frequency of treatment of osteoporosis among postmenopausal women following a fracture. Archives of Internal Medicine 2003, 163(17):2052-2057.

9. Feldstein A, Elmer PJ, Orwoll E, Herson M, Hillier T: Bone mineral density measurement and treatment for osteoporosis in older individuals with fractures: a gap in evidence-based practice guideline implementation. Archives of Internal Medicine 2003, 163(18):2165-2172.

10. Siris ES, Bilezikian JP, Rubin MR, Black DM, Bockman RS, Bone HG, Hochberg MC, McClung MR, Schnitzer TJ: Pins and plaster aren't enough: a call for the evaluation and treatment of patients with osteoporotic fractures. Journal of Clinical Endocrinology \& Metabolism 2003, 88(8):3482-3486.

11. Torgerson DJ, Dolan P: Prescribing by general practitioners after an osteoporotic fracture. Annals of the Rheumatic Diseases 1998, 57(6):378-379.

12. Guidelines for preclinical evaluation and clinical trials in osteoporosis. Geneva: WHO; 1994.

13. Klotzbuecher CM, Ross PD, Landsman PB, Abbott ITA, Berger M: Patients with prior fractures have an increased risk of future fractures: $A$ summary of the literature and statistical synthesis. Journal of Bone and Mineral Research 2000, 15(4):721-739.

14. Wu F, Mason B, Horne A, Ames R, Clearwater J, Liu M, Evans MC, Gamble GD, Reid IR: Fractures between the ages of 20 and 50 years increase women's risk of subsequent fractures. Archives of Internal Medicine 2002, 162(1):33-36.

15. Morris CA, Cabral D, Cheng H, Katz JN, Finkelstein JS, Avorn J, Solomon DH: Patterns of bone mineral density testing: current guidelines, testing rates, and interventions. Journal of General Internal Medicine 2004, 19(7):783-790.

16. Solomon DH, Morris C, Cheng H, Cabral D, Katz JN, Finkelstein JS, Avorn J: Medication use patterns for osteoporosis: an assessment of guidelines, treatment rates, and quality improvement interventions. Mayo Clinic Proceedings 2005, 80(2):194-202.

17. Harrington JT, Broy SB, Derosa AM, Licata AA, Shewmon DA, Harrington JT, Broy SB, Derosa AM, Licata AA, Shewmon DA: Hip fracture patients are not treated for osteoporosis: a call to action. Arthritis \& Rheumatism 2002, 47(6):651-654

18. Majumdar SR, Rowe BH, Folk D, Johnson JA, Holroyd BH, Morrish DW, Maksymowych WP, Steiner IP, Harley CH, Wirzba BJ, et al: A controlled trial to increase detection and treatment of osteoporosis in older patients with a wrist fracture. Annals of Internal Medicine 2004, 141(5):366-373.

19. Eisman J, Clapham S, Kehoe L, Australian BoneCare S: Osteoporosis prevalence and levels of treatment in primary care: the Australian BoneCare Study. Journal of Bone \& Mineral Research 2004, 19(12):1969-1975.

20. Follin SL, Black JN, McDermott MT: Lack of diagnosis and treatment of osteoporosis in men and women after hip fracture. Pharmacotherapy 2003, 23(2):190-198.
21. Marshall $\mathrm{D}$, Johnell $\mathrm{O}$, Wedel $\mathrm{H}$ : Meta-analysis of how well measures of bone mineral density predict occurrence of osteoporotic fractures. BM 1996, 312(7041):1254-1259.

22. Black DM, Cummings SR, Melton L: Appendicular bone mineral and a woman's lifetime risk of hip fracture. Journal of Bone \& Mineral Research 1992, 7(6):639-646

23. Cummings SR, Black DM, Nevitt MC, Browner W, Cauley J, Ensrud K, Genant HK, Palermo L, Scott J, Vogt TM: Bone density at various sites for prediction of hip fractures. The Study of Osteoporotic Fractures Research Group. Lancet 1993, 341(8837):72-75.

24. Storm T, Thamsborg G, Steiniche T, Genant HK, Sorensen OH: Effect of intermittent cyclical etidronate therapy on bone mass and fracture rate in women with postmenopausal osteoporosis. New England Journal of Medicine 1990, 322(18):1265-1271.

25. Black DM, Cummings SR, Karpf DB, Cauley JA, Thompson DE, Nevitt MC, Bauer DC, Genant HK, Haskell WL, Marcus R, et al: Randomised trial of effect of alendronate on risk of fracture in women with existing vertebral fractures. Fracture Intervention Trial Research Group. Lancet 1996, 348(9041):1535-1541.

26. Pols HA, Felsenberg D, Hanley DA, Stepan J, Munoz-Torres M, Wilkin TJ, Qin-sheng G, Galich AM, Vandormael K, Yates AJ, et al: Multinational, placebo-controlled, randomized trial of the effects of alendronate on bone density and fracture risk in postmenopausal women with low bone mass: results of the FOSIT study. Fosamax International Trial Study Group. Osteoporosis International 1999, 9(5):461-468.

27. Harris ST, Watts NB, Genant HK, McKeever CD, Hangartner T, Keller M, Chesnut $\mathrm{CH}$, Brown J, Eriksen EF, Hoseyni MS, et al: Effects of risedronate treatment on vertebral and nonvertebral fractures in women with postmenopausal osteoporosis: a randomized controlled trial. Vertebral Efficacy With Risedronate Therapy (VERT) Study Group. JAMA , 3 1999, 282(14):1344-1352.

28. Ettinger B, Black DM, Mitlak BH, Knickerbocker RK, Nickelsen T, Genant HK Christiansen C, Delmas PD, Zanchetta JR, Stakkestad J, et al: Reduction of vertebral fracture risk in postmenopausal women with osteoporosis treated with raloxifene: Results from a 3-year randomized clinical trial. Journal of the American Medical Association 1999, 282(7):637-645.

29. Chesnut CH, Silverman S, Andriano K, Genant H, Gimona A, Harris S, Kiel D, LeBoff $M$, Maricic M, Miller $P$, et al: A randomized trial of nasal spray salmon calcitonin in postmenopausal women with established osteoporosis: the prevent recurrence of osteoporotic fractures study. PROOF Study Group. American Journal of Medicine, 3 2000, 109(4):267-276.

30. Orwoll E, Ettinger M, Weiss S, Miller P, Kendler D, Graham J, Adami S, Weber K, Lorenc R, Pietschmann $\mathrm{P}$, et al: Alendronate for the treatment of osteoporosis in men. New England Journal of Medicine 2000, 343(9):604-610.

31. Physicians' Guide to Prevention and Treatment of Osteoporosis. [http:// openlibrary.org/books/OL20793907M/Physician\% 27s_guide_to_prevention_and_treatment_of_osteoporosis].

32. Brown JP, Josse RG, Scientific Advisory Council of the Osteoporosis Society of C: 2002 clinical practice guidelines for the diagnosis and management of osteoporosis in Canada. CMAJ Canadian Medical Association Journal 2002, 167(10 Suppl):S1-34.

33. Management of Osteoporosis Edinburgh: Scottish Intercollegiate Guidelines Network; 2003.

34. Compston J, Cooper A, Cooper C, Francis R, Kanis J, Marsh D, McCloskey E, Reid D, Selby P, Wilkins M, et al: Guideline for the diagnosis and management of osteoporosis in postmenopausal women and men from the age of 50 years in the UK. National Osteoporosis Guideline Group (NOGG); 2008.

35. Alendronate, etidronate, risedronate, raloxifene, strontium ranelate and teriparatide for the secondary prevention of osteoporotic fragility fractures in postmenopausal women NICE; 2008

36. McKercher HG, Crilly RG, Kloseck M: Osteoporosis management in longterm care. Survey of Ontario physicians. Canadian Family Physician 2000, 46:2228-2235.

37. Pal B, Morris J, Muddu B: The management of osteoporosis-related fractures: a survey of orthopaedic surgeons' practice. Clinical \& Experimental Rheumatology 1998, 16(1):61-62.

38. Sheehan J, Mohamed F, Reilly M, Perry IJ: Secondary prevention following fractured neck of femur: a survey of orthopaedic surgeons practice. Irish Medical Journal 2000, 93(4):105-107. 
39. Simonelli C, Killeen K, Mehle S, Swanson L: Barriers to osteoporosis identification and treatment among primary care physicians and orthopedic surgeons. Mayo Clinic Proceedings 2002, 77(4):334-338.

40. Kaufman JD, Bolander ME, Bunta AD, Edwards BJ, Fitzpatrick LA, Simonelli C Barriers and solutions to osteoporosis care in patients with a hip fracture. Journal of Bone \& Joint Surgery - American Volume 2003, 85A(9):1837-1843.

41. Jaglal SB, Mclsaac WJ, Hawker G, Carroll J, Jaakkimainen L, Cadarette SM, Cameron C, Davis D: Information needs in the management of osteoporosis in family practice: an illustration of the failure of the current guideline implementation process. Osteoporosis International 2003, 14(8):672-676

42. Taylor JC, Sterkel B, Utley M, Shipley M, Newman S, Horton M, FitzClarence $\mathrm{H}$ : Opinions and experiences in general practice on osteoporosis prevention, diagnosis and management. Osteoporosis International 2001, 12(10):844-848

43. Cuddihy MT, Amadio PC, Melton LJ: Patient barriers to osteoporosis interventions after fracture. Mayo Clinic Proceedings , 3 2002, 77(8):875, author reply 875-876.

44. Higgins J, Altman D: Assessing risk of bias in included studies. In Cochrane Handbook for Systematic Reviews of Interventions Volume Version 5.0.2. Edited by: Higgins J, Green S. The Cochrane Collaboration; 2009:

45. Cochrane Effective Practice and Organisation of care Group. [http://www epoc.cochrane.org].

46. Gardner MJ, Brophy RH, Demetrakopoulos D, Koob J, Hong R, Rana A, Lin JT, Lane JM, Gardner MJ, Brophy RH, et al: Interventions to improve osteoporosis treatment following hip fracture. A prospective, randomized trial. Journal of Bone \& Joint Surgery - American Volume 2005, 87(1):3-7.

47. Feldstein A, Elmer PJ, Smith DH, Herson M, Orwoll E, Chen C, Aickin M, Swain MC, Feldstein A, Elmer PJ, et al: Electronic medical record reminder improves osteoporosis management after a fracture: a randomized, controlled trial. Journal of the American Geriatrics Society 2006, 54(3):450-457

48. Davis JC, Guy P, Ashe MC, Liu-Ambrose T, Khan K, Davis JC, Guy P, Ashe MC, Liu-Ambrose T, Khan K: HipWatch: osteoporosis investigation and treatment after a hip fracture: a 6-month randomized controlled trial. Journals of Gerontology Series A-Biological Sciences \& Medical Sciences 2007, 62(8):888-891.

49. Majumdar SR, Beaupre LA, Harley CH, Hanley DA, Lier DA, Juby AG, Maksymowych WP, Cinats JG, Bell NR, Morrish DW, et al: Use of a case manager to improve osteoporosis treatment after hip fracture: results of a randomized controlled trial. Archives of Internal Medicine 2007, 167(19):2110-2115.

50. Solomon DH, Polinski JM, Stedman M, Truppo C, Breiner L, Egan C, Jan S, Patel M, Weiss TW, Chen YT, et al: Improving care of patients at-risk for osteoporosis: a randomized controlled trial. Journal of General Internal Medicine 2007, 22(3):362-367.

51. Cranney A, Lam M, Ruhland L, Brison R, Godwin M, Harrison MM, Harrison MB, Anastassiades T, Grimshaw JM, Graham ID, et al: A multifaceted intervention to improve treatment of osteoporosis in postmenopausal women with wrist fractures: a cluster randomized trial. Osteoporosis International 2008, 19(12):1733-1740.

52. Majumdar SR, Johnson JA, McAlister FA, Bellerose D, Russell AS, Hanley DA, Morrish DW, Maksymowych WP, Rowe BH, Majumdar SR, et al: Multifaceted intervention to improve diagnosis and treatment of osteoporosis in patients with recent wrist fracture: a randomized controlled trial. CMAJ Canadian Medical Association Journal 2008, 178(5):569-575.

53. Miki RA, Oetgen ME, Kirk J, Insogna KL, Lindskog DM, Miki RA, Oetgen ME, Kirk J, Insogna KL, Lindskog DM: Orthopaedic management improves the rate of early osteoporosis treatment after hip fracture. A randomized clinical trial. Journal of Bone \& Joint Surgery - American Volume 2008, 90(11):2346-2353.

54. Rozental TD, Makhni EC, Day CS, Bouxsein ML, Rozental TD, Makhni EC, Day CS, Bouxsein ML: Improving evaluation and treatment for osteoporosis following distal radial fractures. A prospective randomized intervention. Journal of Bone \& Joint Surgery - American Volume 2008 , 90(5):953-961.

55. Bessette L, Ste-Marie LG, Jean S, Shawn Davison K, Beaulieu M, Baranci M, Bessant J, Brown JP: Recognizing osteoporosis and its consequences in Quebec (ROCQ): Background, rationale, and methods of an anti-fracture patient health-management programme. Contemporary Clinical Trials 2008, 29(2):194-210.

56. Solomon DH, Brookhart MA, Polinski J, et al: Osteoporosis action: design of the healthy bones project trial. Contemporary Clinical Trials 2005, 26(1):78-94

57. Morrish DW, Beaupre LA, Bell NR, Cinats JG, Hanley DA, Harley CH, Juby AG, Lier DA, Maksymowych WP, Majumdar SR, et al: Facilitated bone mineral density testing versus hospital-based case management to improve osteoporosis treatment for hip fracture patients: additional results from a randomized trial. Arthritis \& Rheumatism 2009, 61(2):209-215.

58. Morrison LS, Tobias JH, Morrison LS, Tobias JH: Effect of a case-finding strategy for osteoporosis on bisphosphonate prescribing in primary care. Osteoporosis International 2005, 16(1):71-77.

59. Solomon DH, Finkelstein JS, Polinski JM, Arnold M, Licari A, Cabral D, Canning C, Avorn J, Katz JN, Solomon DH, et al: A randomized controlled trial of mailed osteoporosis education to older adults. Osteoporosis International 2006, 17(5):760-767.

60. Colon-Emeric CS, Lyles KW, House P, Levine DA, Schenck AP, Allison J, Gorospe J, Fermazin M, Oliver K, Curtis JR, et al: Randomized trial to improve fracture prevention in nursing home residents. American Journal of Medicine 2007, 120(10):886-892.

61. Solomon DH, Katz JN, Finkelstein JS, Polinski JM, Stedman M, Brookhart MA, Arnold M, Gauthier S, Avorn J, Solomon DH, et al: Osteoporosis improvement: a large-scale randomized controlled trial of patient and primary care physician education. Journal of Bone \& Mineral Research 2007, 22(11):1808-1815.

62. Bliuc D, Eisman JA, Center JR, Bliuc D, Eisman JA, Center JR: A randomized study of two different information-based interventions on the management of osteoporosis in minimal and moderate trauma fractures. Osteoporosis International 2006, 17(9):1309-1317.

63. Majumdar SR, Lier DA, Beaupre LA, Hanley DA, Maksymowych WP, Juby AG, Bell NR, Morrish DW, Majumdar SR, Lier DA, et al: Osteoporosis case manager for patients with hip fractures: results of a cost-effectiveness analysis conducted alongside a randomized trial. Archives of Internal Medicine 2009, 169(1):25-31.

64. Kastner M, Straus SE, Kastner M, Straus SE: Clinical decision support tools for osteoporosis disease management: a systematic review of randomized controlled trials. Journal of General Internal Medicine 2008, 23(12):2095-2105.

65. Michie S, Fixsen D, Grimshaw JM, Eccles MP: Specifying and reporting complex behaviour change interventions: the need for a scientific method. Implementation Science 2009, 4:40.

doi:10.1186/1748-5908-5-80

Cite this article as: Little and Eccles: A systematic review of the effectiveness of interventions to improve post-fracture investigation and management of patients at risk of osteoporosis. Implementation Science 2010 5:80.

\section{Submit your next manuscript to BioMed Central and take full advantage of:}

- Convenient online submission

- Thorough peer review

- No space constraints or color figure charges

- Immediate publication on acceptance

- Inclusion in PubMed, CAS, Scopus and Google Scholar

- Research which is freely available for redistribution

Submit your manuscript at www.biomedcentral.com/submit
C Biomed Central 